\title{
Wireless Network Virtualization
}

\author{
Xin Wang, Prashant Krishnamurthy, and David Tipper \\ Graduate Telecommunications and Networking Program, University of Pittsburgh, Pittsburgh, USA \\ Email: xiw54@pitt.edu; prashk@pitt.edu; tipper@tele.pitt.edu
}

\begin{abstract}
The virtualization of wired networks and end computing systems has become one of the leading trends in networked information and communication technology (ICT) systems. In contrast relatively little virtualization has occurred in infrastructure based wireless networks, but the idea of virtualizing wireless access is gaining attention as it has the potential to improve spectrum utilization and perhaps create new services. In this paper we survey the state of the current research in virtualizing wireless networks. We define and describe possible architectures, the issues, hurdles and trends towards implementation of wireless network virtualization.
\end{abstract}

Index Terms-wireless virtualization, spectrum sharing, centralized management

\section{INTRODUCTION}

Virtualization refers to the creation of a set of logical architectures using a given set of physical entities, but in a manner that is transparent to the user. For example, a physical server comprised of processors, memory, network interface cards, and storage may be used to create a set of "virtual" servers that all employ the physical hardware, but the users see these virtual servers as separate entities by themselves. The challenge is to allocate the physical entities to the virtual entities in a way that maximizes utilization of the physical entities while providing the required performance to the user. Ideally, such an allocation should be dynamic depending on the needs of users. Further, the allocation process should not be cumbersome or resource intensive by itself. The reasons for virtualization are increased hardware efficiencies, easier migration to newer products or technologies while supporting legacy products, and overall reduced cost of equipment and management. Virtualization of end systems such as servers and cloud computing systems is now widespread and commonplace. Note, that the concept of virtualizing computing systems is actually an old idea [1] originating with IBM during the 1960's, but the concept didn't take off until the economic considerations became the dominant factor. Currently virtualization of computing systems is characterized by three properties: isolation, customization and resource efficiency. That is isolation of users,

\footnotetext{
${ }^{1}$ Manuscript received April 10, 2013; revised April 27, 2013.
} doi: $10.12720 / \mathrm{jcm} \cdot 8 \cdot 5 \cdot 337-344$ customization of services and greater utilization of systems.

Virtualization is well established in wired networks with Virtual Private Networks (VPNs) within service provider networks at various layers (e.g., optical wavelength, MPLS, etc.) common in WANs and MANs and VLANs. Also, Virtual Local Area Networks (VLANs) are widely used in wired enterprise networks. In general the virtualization is achieved by logically partitioning a physical network into virtual networks that share the physical routers/switches/crossconnects, physical links, and bandwidth on each link. The utilization of the physical resources needs to be carefully managed to maintain the quality of service (QoS) and security needs of the users of each virtual network. In the case of WANs and MANs the process of set up/tear down and management of virtual networks is typically tightly controlled by service providers. This has lead to recent efforts on virtual overlay/over-the-top networks that may span multiple service providers and research efforts on providing more generalized virtualization that can be comanaged/set up by users in next generation network architectures.

The motivations for virtualizing wireless networks follow from the observed benefits in a wired network. First, it becomes a natural extension of wired network/end systems virtualization and can potentially enable segregation of traffic (e.g., in terms of QoS, Security) and provide a mechanism to support the popular idea of bring your own device (BYOD) to organizations. Second, spectrum is a scarce resource and virtualization of spectrum has the potential to provide better utilization, making it more efficient for operators. Third, it allows decoupling operators from the cost of infrastructure ownership (capital and operation expenditures) and to also decouple service providers from operators. In such cases, users will simply subscribe to services or applications. The operators just deliver the access service. In other words, this may even decouple users from operators! Lastly, it will likely support the emergence of new services.

When virtualization is applied to wireless networks, things quickly become complicated and large differences with virtualized wired networks occur. Wireless network virtualization includes both infrastructure sharing and spectrum sharing. Furthermore, there are many different topologies for wireless networks (infrastructure and ad hoc, and within ad hoc, single and multi-hop), different spectrum bands ranging from several hundred $\mathrm{MHz}$ to 
several $\mathrm{GHz}$, unlicensed versus licensed spectrum bands, different geographic coverage (wide, metro, local, and personal area) and finally, different mobility requirements. When wireless networks are deployed, the interference that is caused within an administrative unit (e.g., one service provider's network) and across administrative units becomes important. Physical entities in wireless networks can be as diverse as the complex mobility management entity in $4 \mathrm{G}$ cellular networks to inexpensive access points in Wi-Fi networks. Furthermore, the air-interface and bandwidth slices employed by different technologies can be very different. The protocols on the air (access) and in the backbone (core) networks can be very different across technologies. Also, unlike wired networks, users and services can be mobile in wireless networks. Lastly, it is worth noting that governments heavily regulate the basic resource of spectrum and how it can be used.

Thus far, there is no unified vision of what wireless network virtualization means and how it may be achieved. There have been recent attempts to carve out areas where virtualizing wireless networks seems to be possible, albeit in a restricted manner. This work has been motivated by two different activities, namely: (a) the work on dynamic spectrum access and (b) the work on virtualization within a specific technology (e.g., LTE, WiMAX, etc.) for a specific scenario (e.g., infrastructure network, mesh network, etc.).

In this paper, we present our view of wireless network virtualization. We provide some background of recent work in this area in Section II. In Section III, we describe three wireless network virtualization paradigms. Section IV considers the challenges and hurdles in implementation of virtualization in wireless networks. Lastly, Section V concludes the paper.

\section{BACKGROUND}

Recently there has been some work that has started a discussion of wireless network virtualization. We can classify the literature based on whether the origins are rooted in (a) dynamic spectrum access for cellular networks where mobile virtual network operators (MVNOs) are prevalent or (b) based on the technologies considered (e.g., cellular vs. Wi-Fi in infrastructure or ad hoc modes). In this section, we briefly discuss some of this work (see Fig. 1 for a summary).

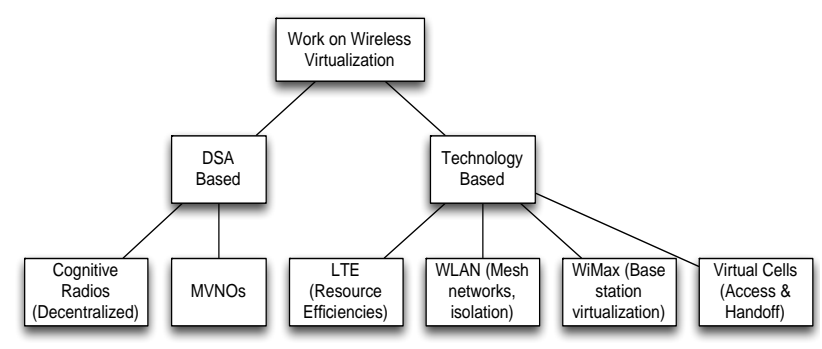

Figure 1. Classification of related work

\section{A. DSA and MVNO Approaches}

\section{1) Work originating in DSA}

Dynamic Spectrum Access (DSA) breaks the hurdle between traditional regulated frequency bands. It creates an open environment for any entities to make use of the available spectrum. Much of the research endeavors related to DSA are in the area of cognitive radio networks. Cognitive radio based networks use a decentralized paradigm in which secondary users sense and access the licensed spectrum bands without interfering with the transmissions of primary users, who operate in the same spectrum in the same geographical area. Like every other decentralized paradigm in wireless networks, collisionavoidance (and hidden terminal issues) is a problem (especially since the interference is at a receiver, which may not be easily detected). Cognitive radios (CRs) have been considered as a promising option to access (licensed) spectrum that is not spatial-temporally utilized. In some ways, the use of CRs with dynamic spectrum access may be viewed as a "type" of spectrum virtualization. In a CR environment, unlicensed or secondary users may sense and access the licensed bands on a negotiated or an opportunistic basis. This is a user-centric paradigm, where the CR does everything in a localized manner. However, it is very difficult to address technical problems such as "hidden nodes" with DSA. Even if a CR user A detects a certain portion of the spectrum available and then starts sending signals, A's transmission may still interfere with radio transmissions of other secondary users or of the primary user since radio propagation is unpredictable as are the locations of other users.

Unlike CR's a centralized spectrum-sharing paradigm that is related to wireless virtualization has appeared recently to address the access problem. In this paradigm, licensed frequency bands can be pooled together in a limited geographical area and may be shared by multiple service providers based on their own interests. The important aspects in a CR-based spectrum sharing approach include spectrum sensing (searching so-called "white spaces" in spectrum where a primary user may not be transmitting), spectrum management (choosing the "best" available spectrum), and spectrum mobility (switching between frequency bands, for e.g., when the primary user starts transmission in a spectrum slice that was previously free). In contrast, in a centralized architecture, all of these aspects are merged into a single issue of "resource scheduling". The central controller gathers channel condition information and each service provider's QoS requirements. Then it assigns the spectrum slices to service providers in each time unit, which is typically short term. If the scheduling is done correctly, such well-scheduled spectrum slices will not face co-channel interference in a given geographical area. The challenge remains as to how this can be combined with frequency-reuse schemes over wider geographical areas and address isolation of users and customization of services at the same time in a scalable fashion. 
To coordinate a large number of CRs, some research papers have integrated brokers into the telecommunication business model [2], [3]. Such dynamic frequency brokers (DFBs) are responsible for allocating frequency bands to radio nodes within their geographic area. Radio nodes submit reports on channel conditions, QoS requirements etc. to DFBs every time interval. DFBs work in a hierarchical manner, with national level DFBs on top of the regional level DFBs. The frequency band allocation is enforced from top to bottom [3]. Bidding procedures (between users and service providers (SPs), or between SPs and spectrum brokers) have been modeled based on game theory in the literature [4]-[6]. In this model, no barriers or obstacles are placed for the utilization of spectrum across the entire bandwidth. The spectrum pool is drained based on users demand. In other words, the wireless network evolves into a virtual environment with the presence of DFBs. Spectrum users run their operations without knowing the underlying architecture.

However, virtualization of wireless networks, as described later in this paper, is an even broader concept than the DFB system.

2) Mobile virtual network operator approaches

A Mobile Virtual Network Operator (MVNO) is a special network operator who leases radio access from a host mobile network operator (MNO). An MVNO can be viewed as a special implementation of wireless virtualization. The strict definition of an MVNO differs from country to country [7]. Typically a MVNO leases spectrum from one or more MNOs, and connects its own subscribers to its own switching center. Alternatively, the network operated by an MVNO may also be connected to the MNO's networks that have agreements with the MVNO. The key distinction between a MVNO and a MNO is that an MVNO does not own any spectrum and radio access networks. In some countries, regulators may require MNOs to open the networks to MVNOs to enhance competition. On the other hand, MNOs may also make voluntary agreements to engage with MVNOs to derive some benefits. For instance, MVNOs can reach or test new market segments, utilize spare network capacity, and introduce new services which can supplement existing services provided by an MNO [8].

Although the MVNO concept may bring about much needed service differentiation to a cellular network, it is still not a model of complete virtualization for the overall wireless network. MVNOs lease a fixed amount of resources (e.g., bits transferred) from MNOs in a longterm mostly static fashion. Currently, the radio resources in the access network are not dynamically shared among multiple MVNOs or across MNOs in a fine granularity. This approach has been suggested with LTE as discussed in the next section.

\section{B. Technology Oriented Approaches}

1) LTE based: The use of LTE for virtualization has been recently explored in the literature. The idea is similar to router/switch virtualization in wired networks. The work in [9]-[11] proposed an entity called a "Hypervisor" on top of the physical layer in the base stations in LTE (called e-NodeB's or eNBs). The hypervisor virtualizes the eNB into a number of virtual eNBs (each of which is managed by a virtual operator). The hypervisor also allocates the air interface resources (called physical resource blocks or PRBs in LTE) among multiple virtual eNBs.

Virtual operators share the LTE spectrum based on QoS criteria and give feedback to the hypervisor in each time unit. The hypervisor collects information from individual virtual eNB stacks, such as user channel conditions, traffic loads, priorities, QoS requirements and information related to the contract of each virtual operator [9]. Based on this information, the hypervisor can schedule the air interface resources among multiple virtual networks every time unit. Different configuration methods [9]-[11] can be used to complete the scheduling. When the budget of spectrum allocation tightly follows the traffic load, multiplexing gains are reported based on simulations of such virtual networks.

2) WLAN based: The virtualization of WLAN access points has been considered in [12]. Rather than pooling spectrum, this work considers resource partitioning of limited spectrum in an optimal and fair way. To this end, the authors manipulate in each virtual WLAN, the contention window in the CSMA/CA IEEE 802.11 based medium access control protocol. Also, a "SplitAP" architecture was proposed in [13] providing airtime fairness for group of WLAN users. A single physical access point can emulate multiple virtual access points associate with corresponding users. Virtual device design usually asks for three fundamental principles - abstraction, programmability and isolation. Abstraction allows one physical structure to split into multiple virtual ones. Programmability controls the virtual access points. Isolation makes sure that the system performance for each virtual network is not affected by other virtual networks. The complexity of embedding a virtual network over a physical wireless mesh network is studied in [14].

3) WiMAX based: A "virtual base station" design has been proposed by applying the three fundamental principles for virtualization mentioned previously (resource efficiency, isolation, and customization). The additions and modifications needed for virtualizing WiMAX base stations are addressed in [15]. The virtualized base station performs frame switching at the MAC layer. Meanwhile, the isolation mechanism improves the aggregate throughput significantly for different classes of users. Another general framework to virtualize WiMAX networks comes with an optimal slice scheduler aiming at isolation, 
customization and efficient resource utilization [16]. Concave utility functions are defined and maximized using a simple weighted solution. Although the isolation and customization can be achieved using the weighted fairness algorithm on a long-term basis, it becomes hard to ensure an effective data rate for each user in every time unit. Further, operations over multi-cell and wider geographical areas have not been studied yet.

4) Mobile platform based: The trend of massive smart phone usage indicates that the primary platform for mobile users in the future would be small devices, the computing capabilities of which are limited by the battery and processors. Migrating computation from small mobile devices such as tablets to desktops or laptops, which have more resources and processing capabilities, is discussed as a virtualization topic as well. The computation migration becomes important for the development and usage of some complex mobile apps. This type of virtualization requires hardware support, like allowance for operating systems existence and software virtualization. The work in [17] introduced a usage model, which offloads computation between virtual machines using a fast local wireless network. While this is not necessarily wireless network virtualization, we include it here as it is related to the end devices, but do not elaborate on it further.

5) Access selection based: The above-mentioned technology-oriented virtualization related work considers s either models for networks or specific platforms. Network virtualization mostly happens at the MAC layer on a single network component. The mobile computation migration can be seen as a transition at the application layer, although it has certain hardware requirements. Recently, a new term "virtual cell" has been proposed in [18]. A "virtual cell" is a limited geographical area with homogeneous radio conditions. Differing coverage of different wireless access points is no longer an issue. Instead, the network chooses the proper wireless interface for mobile users along their projected route. In [18], the handover transition along the projected route is modeled for maximizing the reliability of a constant wireless connection.

\section{WIRELESS NETWORK VIRTUALIZATION PARADIGMS}

Generally, network virtualization, irrespective of whether it is in wired or wireless environments, can be viewed as splitting the entire system. It is possible to view the network as being composed of Infrastructure Providers or InP's that create and manage only the infrastructure (e.g., base stations, MMEs, S-Gateways, APs, spectrum, etc.) and Service Providers or SPs, which actually provide various services to subscribers. The resources that belong to one or more InPs are virtualized and split into slices. A SP requires a minimum of one slice of the resources from InPs and provides end-to-end services to end-users, without knowing the underlying physical architecture of the InP. After splitting the resources into slices, each slice creates an illusion that it is an entire system by itself. This "slice" system consists of its own (virtualized) core network and (virtualized) access network corresponding to the wired slice and the wireless slice, respectively.

In recent research work, various analytical and experimental models have been proposed to depict wireless virtualization and evaluate virtual architectures [5], [6], [9]-[11], [19], [20]. On the one hand, work that focuses on market profit views a virtual wireless network simply as a spectrum pool with hierarchical DFB management as described previously. In such cases, two types of interactions are studied - between users and SPs or between SPs and InPs. Such interactions usually are modeled as stochastic games. The existence of the Nash equilibrium [5] can result in an optimum price for spectrum. On the other hand, research that focuses on implementation of wireless virtualization pick a particular platform like LTE or Wi-Fi. Such works consider case studies and run simulations to evaluate the technical benefits of virtualization. Compared to the work that looks at spectrum pools, the related work on the technical implementation is limited. Also, a few works aim at virtualizing one $\mathrm{BS}$ to fit the requirements of multiple MVNOs. Some optimization techniques like weighted slice allocation are integrated within the physical BS to create opportunities for MVNOs. MVNOs can then customize their own virtual BSs [20]. However, the MVNOs will need to be able to virtualize the backhaul network and it's components (signaling, mobility management, security functions, localization, etc.) as well. In a nutshell, even the understanding of what wireless virtualization means is not clear in the literature.

Inspired by the different degrees of virtualization, this paper proposes three paradigms for wireless network virtualization employing the idea of InPs and SPs, namely: (1) universal, (2) cross-infrastructure, and (3) and limited intra-infrastructure. As shown in Fig. 2 the three paradigms from (3) limited intra-infrastructure towards (1) universal have progressively more virtualization.

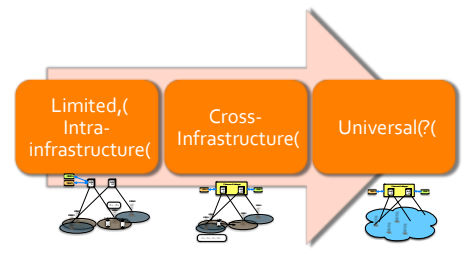

Figure 2. Paradigms of wireless virtualization

\section{A. Universal Virtualization}

A grand vision view of wireless network virtualization is to make no assumptions whatsoever about InPs or SPs. 
This view of wireless network virtualization looks at the whole path of radio access as an "unbundled cloud" where virtualization is pervasive. The cloud is comprised of heterogeneous base stations (macro-cells, pico-cells, and femto-cells, relays, and other kinds of points of access as well as wired backbones) that are transparent to the user [6]. It is the responsibility of a SP of a specific service to choose a package of network components, links, and spectrum and the SP configures them in the way it desires. Ideally, this could happen dynamically in an ondemand type fashion. For example, to support a specific application such as one that involves extremely low power transmissions at low rates with not very stringent delay constraints, the network components to be used may be femto-cells using a small slice of spectrum or even sensor relays that use multiple hops to a destination. This "cloud" like virtualization has complicated management, control and economic issues that have not been considered in the literature. For example, how much and what type of management capabilities are given to a SP on InP system, how can isolation of SPs be enforced and how can mandated/regulated services like E-911 localization be ensured are open problems.

\section{B. Cross-infrastructure Virtualization}

In this paradigm we assume that wireless virtualization is possible across InPs (inter-InP) and within InPs. This enables all of the InPs in a geographical area to allow their network resources to be shared across SPs. A simplified example is depicted in Fig. 3. In this example, base stations (BS) 1 and 2 belong to InP 1 while BSs 3 and 4 belong to InP 2. Two SPs are in the system SP A and SP B. A centralized management has to be implemented to ensure the co-operation and isolation between InPs (for this purpose, an entity named "Resource Manager" is added on top of the InPs). Notice that an InP might have bandwidth slices in various frequency bands that support multiple radio access technologies (RATs) such as, GSM, UMTS and LTE. Inter-InP virtualization allows spectrum sharing between different InPs, SPs, and different RATs. InPs that cover the same region (in Fig. 3, BSs 1 \& 3 for example) provide their physical resources to SPs. SPs are allocated specific resources based on their requirements, every specific time unit. Not only are the radio resources shared among different SPs, but also the nodes and links, which connect the access network to the core network. These backhaul nodes and links should be shared in a virtualized fashion. There are no clear boundaries between multiple network infrastructures belonging to different InPs. It is as if all the resources are in the same pool for SPs to employ (e.g., in Fig. 3, frequencies $f_{1 A}, f_{1 B}$, $\mathrm{f}_{3 \mathrm{~A}}, \mathrm{f}_{3 \mathrm{~B}}$ are in the same pool). SPs might choose the resource with the best quality or with the lowest price. However, inter-InP wireless virtualization has strict coverage/interference requirements. The coverage of InPs should either completely overlap or there has to be a way of determining what BSs from which InP covers what part of a geographical area. Otherwise there may be "service holes" when users enter an area which is not covered by a set of InPs used by an SP. Due to the limited wireless coverage of each cell, this virtualization design might be more suitable for certain areas (e.g., urban) that have highly overlapping multiple cells from several InPs.

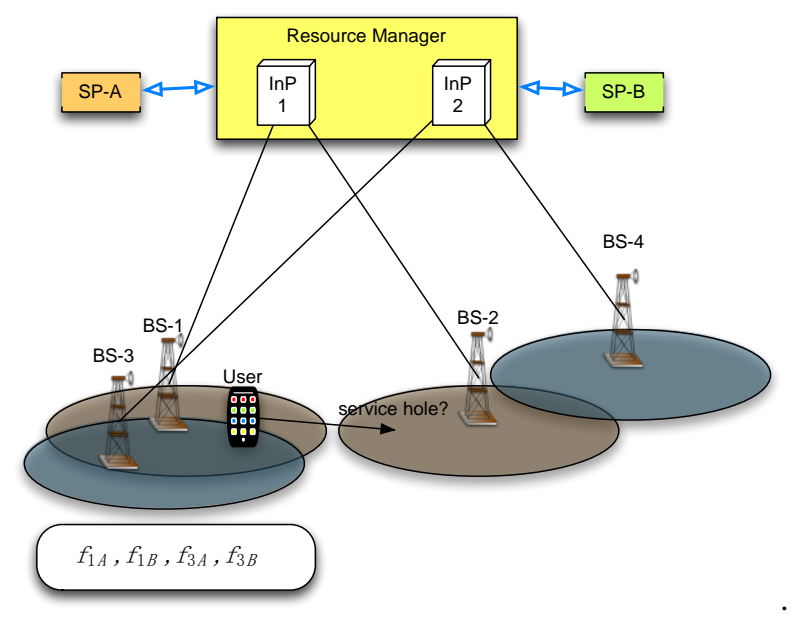

Figure 3. Cross-infrastructure wireless network virtualization

To design an appropriate cross-infrastructure virtualization strategy, several factors have to be taken into account, such as the entire network architecture, the QoS promised by each SP, mobility management and the spatial-temporal variations of traffic, cross InP signaling and location tracking. Hence, for cross-infrastructure wireless virtualization, a completely centralized management may be preferable. A well-designed centralized strategy will have a higher probability of bringing significant improvement to the network utilization, reliability, and quality of service. But a bad strategy might encroach upon the reserved resources of some SP, and such an SP may not be able to ensure a level of QoS for its users, especially highly mobile users and the ones at the edges of coverage due to poor channel quality or excess interference.

\section{Limited Intra-infrastructure Virtualization}

Limited wireless virtualization in our view considers only virtualization within a single InP, which may have spectrum that is used by different RATs. Spectrum sharing occurs between SPs and across RATs. For a given cell, we can think of a single InP that can manage its resources and make decisions to allocate them to various SPs. The multiplexing gains are likely to be lower than those possible with a cross-infrastructure strategy as there may be InPs with demand from SPs that is greater than they can meet, while other InPs have resources that are not being completely utilized. Limited virtualization can be described by the example shown in Fig.4. In cell 1 of a cellular system, two SPs A and B lease a certain amount of resources from BS 2 in each time interval. BS 2 is virtualized and in charge of the spectrum $f_{2 A}$ and $f_{2 B}$ allocated to SPs. This is similar to some work done in BS hardware virtualization (e.g., [15]) described previously. 
Every SP can be viewed as a virtual operator (VO) with time-varying resources based on factors such as its own requirement, the amount of money it is willing to pay for resources, fairness, and other InP policies. In some sense, this is similar to the single-level DFB structure where we can consider the InP as a DFB that assigns spectrum to nodes in its region and SPs as those nodes.

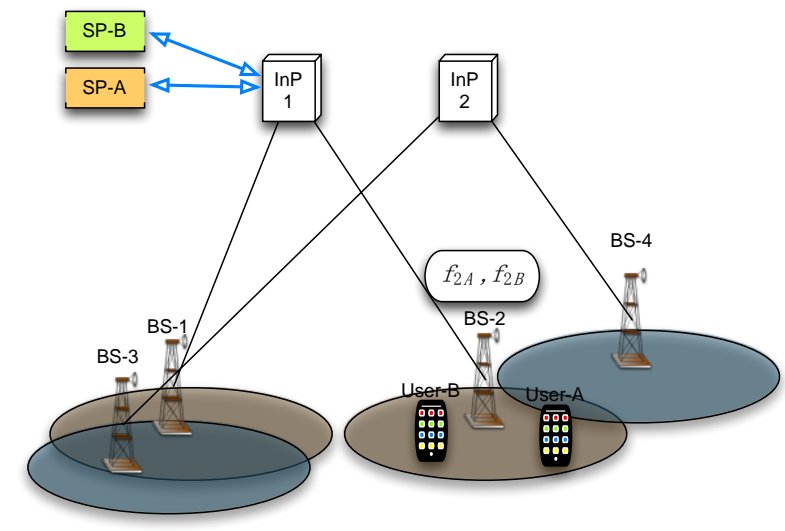

Figure 4. Limited intra-infrastructure wireless network virtualization

Besides scheduling, this paradigm could work in another way as a decentralized spectrum sharing behavior that now exists in cellular networks over the last several years namely that of tiered networks. In some literature, this idea corresponds to the overlay-underlay networks. In this architecture, the system takes advantage of the differences of coverage and radio access conditions between InPs. Small cells embedded in large cells may universally reuse their frequency bands. However, the overlay network formed by Pico/Femto cells needs to be self-organizing by evaluating the cross-tier SINR. In wireless virtualization, cells are all coordinated under central control.

\section{ISSUES FOR DISCUSSION}

There are several challenges that arise irrespective of the wireless network virtualization paradigm adopted (although the specifics are likely to vary in the degree of complexity). As an extension of wired network virtualization, technical challenges in terms of instantiation, operation and management of wireless network virtualization need to be better explored [21]. Most existing work focuses on spectrum allocation models, for example, some theories like auction game winner determination problems are used to model spectrum allocation [5], [6]. A few experiments have been done on hardware testbeds [22], [23]. However, important issues like interfacing, signaling, mobility management, isolation, customization and enforcement have not had much attention. In this subsection, these issues are discussed as challenges mapped from the wired perspective presented in [21].

1) Interfacing: Wired virtualization requires virtual networks to express their needs in terms of virtual nodes and virtual links in a standard specification language [21]. In wireless virtualization, SPs require radio resources (bandwidth, power, interference) from one or more InPs. Since various RATs might be used by SPs on the same InP, a well-defined common interface is a must for InPs to understand the radio resources required by SPs. Furthermore, with multiple InPs, the need for a standard language to express explicit sharing information to each other arises. The communication between SPs and between endusers and SPs also needs to be standardized.

2) Signaling and bootstrapping: A SP must have network connectivity to one or more InPs in order to issue its requests before a virtual network is created. Signaling must be handled properly (in terms of delays and reliability) to enable the InPs or the hypervisor to enable configuring the network it supports. A bootstrapping capability to allow SPs to customize the virtual resources allocated to them is needed. Wireless virtualization may need out-of band wired or wireless communications for these functions. If all of the spectrum is virtualized, at least a piece of it may need to be dedicated for signaling and bootstrapping unless wired links exist to handle these functions.

3) Resource allocation: A well known wired virtual network problem is how to embed a virtual network in a physical network (i.e., what nodes, links, and resources should be picked) [21], and it is also important in wireless virtualization [14]. Embedding of virtual networks, with constraints on resources or requirements, can be reduced to an NP-hard optimization problem. In market-oriented analyses, the problem usually aims to maximizing the revenue of each InP with finite spectrum and SPs QoS requirements as constraints [6]. In crossinfrastructure virtualization, constraints such as finite radio resources, SPs QoS requirements, and different InPs policies need to be included in the problem.

4) Resource Discovery: In order to allocate resources to SPs, InPs or hypervisors should be aware of the available radio resources of the wireless network. Coordination among InPs should be done before each InP assigns its resources to SPs. InPs may need to reserve some resources for themselves in which case the InPs need to determine what radio resources to keep and how much they are willing to share. Resource discovery and allocation create another important issue for network management the transmission time interval (TTI) or time unit between each discovery and allocation of resources. It is obvious that the cost will be astounding if the period is short. But a low update frequency (e.g., monthly SLAs) might drag the network back to the traditional static architecture. 
5) Isolation: The performance of wireless networks is much more sensitive to interference then wired networks, which makes isolation between different users or SPs crucial. In [20], a slice isolation engine (SIE) is used to limit slice traffic irrespective of the clients and services classes agreed before. Another way to deal with isolation is to embed it to the spectrum allocation problem. It can be considered as a constraint in terms of the particular distance between paired spectrum channels [6] for frequency duplexed systems.

6) Mobility management: Mobile users should be able to smoothly switch to their contracted SP. An even better scenario is that users might access any SP either offering the best QoS or the lowest cost in that location. Wireless virtualization facilitates this mobility management through spectrum/infrastructure sharing and protocols between SPs and InPs to ensure that users can access the most appropriate SP.

7) System operation: Wireless virtualization may require all InPs to share their physical resources. If the coverage of several InPs overlaps, or the demand is low, it may be possible to save cost by carefully shutting down some of the BSs and sharing the resources of the other BSs. BSs may need extra hardware and software enablers to adapt to the increased spectrum/RAT capabilities. Such system operations have to be reconciled with resource discovery, allocation, isolation, etc.

\section{A. Limitations of Wireless Virtualization}

1) Finite resources: Unlike cloud computing, the economies of scale that makes virtualization a viable model may not be always applicable to the wireless domain. Coverage in rural areas is often a problem. A smaller number of BSs with limited capabilities in rural areas may not leave enough resources remaining to be shared making virtualization meaningless. Even though the amount of spare resources may be larger than in urban areas, the spread is uneven in geographic terms. Furthermore, spectrum is a regulated resource that cannot easily added to a specific geographic location in contrast to cloud computing where additional computing resources can be quickly added.

2) End device: Wireless virtualization may require end devices to be adapted to enable them to access a broader range of carrier frequencies. Flexible spectrum sharing needs enablers, for instance, frequency agile broadband radios and direct conversion architectures. An end device needs to be equipped with hardware to enable itself to access the entire frequency band. Software to compute the spectrum sharing algorithms should also be available.

\section{B. Non-technical Challenges}

Technically, it appears that the potential multiplexing gains and better spectrum utilization are good reasons for wireless network virtualization. However, wireless network virtualization is unlikely to happen in practice and may suffer the same fate as many other promising but unsuccessful technologies without a good economic rationale and a friendly regulatory environment. One of the few papers that illustrate a use-case for a (limited) virtualized wireless network is the work in [24], which uses enterprise cloud access by mobile devices as a motivating example. In terms of regulation, the ownership of spectrum, physical infrastructure, and provision of services will likely have to be unbundled. It is unlikely that legacy service providers will be willing to easily share their resources unless strong economic and regulatory reasons arise. Furthermore many technoeconomic issues need to be resolved, such as how should spectrum contributions from different InPs be evaluated and scored in common pool since spectrum bands are not entirely fungible [25]. Additionally, for all of the useful spectrums to be virtualized, one-way broadcast communications will also have to be considered and the support for legacy devices carefully examined.

\section{CONCLUSION}

Providing virtualization in wireless networks is a promising concept that has the potential to relieve spectrum congestion and open new services. In this paper we discussed three paradigms for virtualizing wireless networks: (1) universal, (2) cross-infrastructure and (3) limited intra-infrastructure. The paradigms vary in the degree of virtualization and infrastructure sharing. Each paradigm incurs technical and non-technical hurdles that must be overcome before wireless virtualization becomes a widespread technology. These challenges require careful design and evaluation for wireless network virtualization to be come a reality.

\section{REFERENCES}

[1] C. Kalmanek, "The essential elements of successful innovation," ACM Computer Communications Review, vol. 42, no. 2, pp. 105109, April 2012.

[2] T. Maseng and T. Ulversoy, "Dynamic frequency broker and cognitive radio," The IET Seminar on Cognitive Radio and Software Defined Radios: Technologies and Techniques, September 2008.

[3] G. Isiklar and A. Bener, "Brokering and pricing architecture over cognitive radio wireless networks," in Proc. IEEE CCNC, January 2008.

[4] M. M. Buddhikot, P. Kolodzy, S. Miller, K. Ryan, and J. Evans, "Dimsumnet: New directions in wireless networking using coordinated dynamic spectrum," in Proc. IEEE WoWMoM, 2005, pp. 78-85.

[5] F. Fu and U. C. Kozat, "Wireless network virtualization as a sequential auction game," in Proc. IEEE Infocom, 2010.

[6] T. K. Forde, I. Macaluso, and L. Doyle, "Exclusive sharing and the virtualization of the cellular network," in Proc. IEEE Symposium on Dynamic Spectrum Access Networks, 2011. 
[7] B. W. Kima and S. H. Seol, "Economic analysis of the introduction of the MVNO system and its major implications for optimal policy decisions in Korea," Telecommunications Policy, vol. 31, no. 5, pp. 290-304, 2007.

[8] Finnish Ministry of Transport and Communications, "MVNO pricing structures in Finland," Edita Publishing, Helsinki, 2005.

[9] Y. Zaki, L. Zhao, A. Timm-Giel, and C.Gorg, "A novel LTE wireless virtualization framework," in Proc. Second International ICST Conference on Mobile Networks And Management, 2010.

[10] Y. Zaki, L. Zhao, A. Timm-Giel, and C. Gorg, "Lte wireless virtualization and spectrum management," in Proc. Third Joint IFIP Wireless and Mobile Networking Conference, Oct 2010.

[11] Y. Zaki, L. Zhao, C.Gorg, and A. Timm-Giel, "LTE mobile network virtualization," Mobile Networks and Applications, vol. 16, no. 2, pp. 424-432, August 2011.

[12] A. Banchs, P. Serrano, P. Patras, and M. Natkaniec, "Providing throughput and fairness guarantees in virtualized wlans through control theory," Mobile Networks and Applications, vol. 17, no. 4, pp. 435-446, August 2012.

[13] G. Bhanage, D. Vete, I. Seskar, and D. Raychaudhuri "SplitAP: Leveraging wireless network virtualization for flexible sharing of WLANs." in Proc. IEEE Global Telecommunications Conference, 2010.

[14] D. Yun and Y. Yi, "Virtual network embedding in wireless multihop networks," in Proc. ACM Conference on Future Internet Technologies, June 13-15 2011.

[15] I. S. Bhanage, Gautam, and D. Raychaudhuri., "A virtualization architecture for mobile WiMAX networks." ACM SIGMOBILE Mobile Computing and Communications Review, vol. 15, no. 4, pp. 26-37, 2012.

[16] R. Kokku, R. Mahindra, H. Zhang, and S. Rangarjan, "NVS: A virtualization substrate for WiMAX networks." In Proc. $16^{\text {th }} \mathrm{ACM}$ Annual International Conference on Mobile Computing and Networking, Sept., 2010.

[17] S. Sud, R. Want, T. Pering, K. Lyons, B. Rosario and M. Gong, "Dynamic migration of computation through virtualization of the mobile platform." Mobile Networks and Applications, vol. 17, no. 2, pp. 206-215, 2012.

[18] E. Folstad and B. Helvik, "Optimizing service continuity in a multi operator multi technology wireless environment." in Proc. $9^{\text {th }}$ International Conference on Design of Reliable Communication Networks, March 4-7, 2013.

[19] H. Anouar, C. Bonnet, D. Camara, F. Filali, and R. Knopp, “An overview of open air interface wireless network emulation methodology," ACM Sigmetrics Performance Evaluation Review, vol. 36, no. 2, September 2008.

[20] G. Bhanage, I. Seskar, R. Mahindra, and D. Raychaudhuri, "Virtual basestation: Architecture for an open shared wimax framework," in Proc. $2^{\text {nd }}$ ACM SIGCOMM Workshop on Virtualized Infrastructure Systems and Architectures, September, 2010.

[21] N. M. K. Chowdhury and R. Boutaba, "A survey of net-work virtualization," Computer Networks, vol. 54, pp. 862-876, 2010.

[22] Y. He, J. Fang, J. Zhang, H. S. K. Tan, and Y. Zhang, "MPAP: Virtualization architecture for heterogeneous wireless APs," ACM Computer Communications Review, Jan 2011.
[23] S. Singhal, G. Hadjichristofi, I. Seskar, and D. Raychaud- huri, "Evaluation of uml based wireless network virtual- ization," in Proc. Next Generation Internet Networks, 2008.

[24] A. Baliga, X. Chen, B. Coskun, G. de los Reyes, et al., "Vpmnvirtual private mobile network towards mobility-as-a-service," in Proc. ACM $2^{\text {nd }}$ Workshop on Mobile Cloud Computing and Services, 2011.

[25] M. Weiss et al., "When is electromagnetic spectrum fungible," in Proc. IEEE Symposium on Dynamic Spectrum Access Networks, 2012.

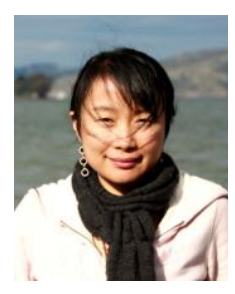

Xin Wang is currently a $\mathrm{PhD}$ student in the Telecommunications and Networking Program, University of Pittsburgh, Pittsburgh, PA. She received her MS degree from Communication and Information department of Shanghai University and BS degree in EE from Shanghai Normal University, both in Shanghai, China. She has one year working experience as a hardware engineer in the Radar Research Institution. She interned in Alcatel-Lucent Bell Lab working on LTE protocol in 2009, Shanghai, China. Her research interests lie in wireless network planning/design, future wireless communication technologies and physical layer security.

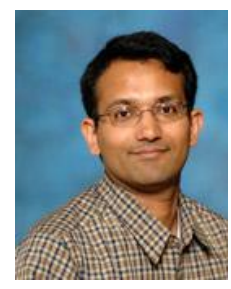

Prashant Krishnamurthy is an associate professor in the School of Information Science, University of Pittsburgh, Pennsylvania, where he regularly teaches courses on wireless networks and cryptography. From 2000 to 2005, he also served as the chair of the IEEE Communications Society Pittsburgh Chapter. His research interests include wireless network security, wireless data networks, position location in indoor wireless networks, and radio channel modeling for indoor wireless networks. He is a member of the IEEE.

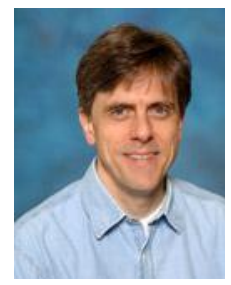

David Tipper is the Director of the Graduate Telecommunications and Networking Program and a faculty member at the University of Pittsburgh, Pittsburgh, PA. He is a graduate of the University of Arizona (Ph.D. EE, MS SIE) and Virginia Tech (BS EE). His current research interests are survivable networks, performance analysis techniques, wireless/wired network design and information assurance. Professor Tipper's research has been supported by grants from various government and corporate sources such as NSF, DARPA, NIST, IBM, ARO and AT\&T. Professional activities include serving as the General Chair of the 7th Design of Reliable Communication Networks Workshop (DRCN2009) and coguest editor of a special issue of the journal Telecommunication Systems on Reliable Networks Design and Modeling which appeared in 2013. He is the co-author of the textbook The Physical Layer of Communication Systems, which was published by Artech House in 2006. Also, he is the co-editor and a contributor to Information Assurance: Dependability and Security in Networked Systems, which was published by Morgan Kaufmann in 2008 